\title{
Multivariate analysis of factors associated with first-pass success in blind placement of a post-pyloric feeding tube: a retrospective study
}

Kohei Kurisawa ${ }^{1 \dagger}$, Masashi Yokose ${ }^{1^{* \dagger}} \mathbb{0}$, Hiroyuki Tanaka' ${ }^{1}$ Takahiro Mihara ${ }^{1,2}$, Shunsuke Takaki ${ }^{1}$ and Takahisa Goto ${ }^{1}$

\begin{abstract}
Background: Trans-jejunal nutrition via a post-pyloric enteral feeding tube has a low risk of aspiration or reflux; however, placement of the tube using the blind method can be difficult. Assistive devices, such as fluoroscopy or endoscopy, are useful but may not be suitable for patients with hemodynamic instability or severe respiratory failure. The aim of this study was to explore factors associated with first-pass success in the blind placement of post-pyloric enteral feeding tubes in critically ill patients.
\end{abstract}

Methods: Data were obtained retrospectively from the medical records of adult patients who had a post-pyloric enteral feeding tube placed in the intensive care unit between January 1, 2012, and December 31, 2018. Logistic regression analysis was performed to assess the association between first-pass success and the independent variables. For logistic regression analysis, the following 13 variables were defined as independent variables: age, sex, height, fluid balance from baseline, use of sedatives, body position during the procedure, use of cardiac assist devices, use of prokinetic agents, presence or absence of intestinal peristalsis, postoperative cardiovascular surgery, use of renal replacement therapy, serum albumin levels, and position of the greater curvature of the stomach in relation to spinal levels L1 - L2.

Results: Data obtained from 442 patients were analyzed. The first-pass success rate was $42.8 \%(n=189)$. Logistic regression analysis demonstrated that the position of the greater curvature of the stomach cephalad to $L 1-L 2$ was only associated with successful placement (odds ratio for first-pass success, 0.62; 95\% confidence interval: 0.40 - 0.95).

Conclusions: In critically ill patients, the position of the greater curvature of the stomach caudal to L1 - L2 may be associated with a lower first-pass success rate of the blind method for post-pyloric enteral feeding tube placement. Further studies are needed to verify our results because the position of the stomach was estimated by radiographs after enteral feeding tube placement.

\footnotetext{
*Correspondence: yokose_p12@yahoo.co.jp

${ }^{\dagger}$ Kohei Kurisawa and Masashi Yokose contributed equally to this

manuscript as co-first authors

1 Department of Anesthesiology and Critical Care Medicine, Yokohama

City University Graduate School of Medicine, 3-9 Fukuura, Kanazawa-ku,

Yokohama 236-0004, Japan

Full list of author information is available at the end of the article
}

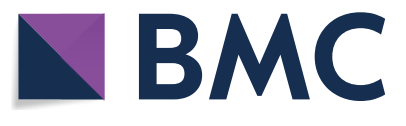

(c) The Author(s) 2021. Open Access This article is licensed under a Creative Commons Attribution 4.0 International License, which permits use, sharing, adaptation, distribution and reproduction in any medium or format, as long as you give appropriate credit to the original author(s) and the source, provide a link to the Creative Commons licence, and indicate if changes were made. The images or other third party material in this article are included in the article's Creative Commons licence, unless indicated otherwise in a credit line to the material. If material is not included in the article's Creative Commons licence and your intended use is not permitted by statutory regulation or exceeds the permitted use, you will need to obtain permission directly from the copyright holder. To view a copy of this licence, visit http://creativecommons.org/licenses/by/4.0/. The Creative Commons Public Domain Dedication waiver (http://creativeco mmons.org/publicdomain/zero/1.0/) applies to the data made available in this article, unless otherwise stated in a credit line to the data. 
Trial registration: University Hospital Medical Information Network Clinical Trials Registry (UMIN000036549; April 20, 2019).

Keywords: Blind placement, Enteral feeding tube, Intensive care unit, Post-pyloric enteral nutrition, Stomach, Greater curvature

\section{Background}

Critically ill patients admitted to the intensive care unit (ICU) are a high-risk group for malnutrition, with a reported prevalence ranging from 38 to $78 \%$ [1]. Malnutrition is associated with muscle atrophy, prolonged ventilation, longer ICU stays, and increased risk of infection and mortality [2-5]. Enteral nutrition is superior to intravenous nutrition in terms of the incidence of infection $[6$, 7], length of hospital stay [8], and medical costs $[9,10]$. Early initiation of enteral nutrition is also recommended to preserve endothelial cell structure and secretory function, and to maintain immunity [11-14]. Post-pyloric enteral nutrition decreases the incidence of respiratory complications compared to trans-gastric feeding $[15,16]$, and it is suitable for patients receiving sedatives or muscle relaxants, or those who cannot tolerate elevation of the head of the bed.

Methods of post-pyloric placement of enteral feeding tubes (EFTs) include endoscopy, fluoroscopy, ultrasound assistance, and electromagnetic guidance. However, EFTs can also be placed blindly without the aid of assistive devices. Fluoroscopy and endoscopy have higher success rates [17-19]; however, they may not be suitable for patients with hemodynamic instability or severe respiratory failure who cannot be transported outside the ICU. Blind placement at the bedside is commonly used in critically ill patients because it is easy, minimally invasive, and inexpensive. However, there is a considerable risk of failure in placing the tube in the correct position, which may lead to delays in enteral nutrition. To our knowledge, only a few studies have investigated the risk factors associated with blind placement difficulties [20].

In this study, we aimed to explore the factors associated with first-pass success of blind placement of post-pyloric EFTs.

\section{Methods}

This retrospective observational study was conducted in accordance with the principles of the Declaration of Helsinki. The study design was approved by the Ethics Board of Yokohama City University Hospital, Yokohama, Japan (approval number: B181000027; November $22,2018)$. The study was registered with the University Medical Information Network Clinical Trials Registry (UMIN000036549; April 19, 2019; principal investigator, Masashi Yokose) before data collection. The Ethics Board waived the requirement to obtain written informed consent owing to the retrospective nature of the study. Consecutive subjects aged $\geq 20$ years who underwent blind placement of a post-pyloric EFT in the ICU of Yokohama City University Hospital from January 1, 2012, to December 31, 2018, were included in this study. The exclusion criteria were a preexisting EFT upon ICU admission, enteric fistula or gastrostomy, and a history of upper gastrointestinal surgery.

\section{Standard procedure for blind EFT placement}

We used an EFT with a stylet (Kangaroo ${ }^{\mathrm{TM}}$ New Enteral Feeding Tube: Covidien Japan; Tokyo, Japan) in all patients. The size of the EFT was selected from 8 to 12 French according to the patient physique. The use of prokinetic agents was permitted when the attending physician felt it was clinically necessary. The right lateral position was selected when the patient's condition permitted; however, this could be changed at the discretion of the physician performing EFT placement. The EFT was inserted through the nose or mouth and was advanced until the tip was presumed to be in the stomach $(40-65 \mathrm{~cm})$, which was confirmed by the sound of the gas over the stomach when $5-10 \mathrm{~mL}$ of air was forcibly injected through the tube. The tube was then slowly advanced a few centimeters at a time. Advancement of the tube was confirmed by releasing the hand after each push of the tube and ensuring that the tube stayed in position. If it was judged that the tube had not advanced, the tube was withdrawn about $5 \mathrm{~cm}$ and advanced again. To estimate the position of the EFT tip, air was forcibly injected through the tube to determine the strongest point of the sound. When the high-pitched sound was most audible in the patient's right lateral abdomen, and the insertion length was approximately $25-30 \mathrm{~cm}$ from the point where it was determined to be in the stomach, $20 \mathrm{~mL}$ of water or air was injected through the EFT, and the tube was aspirated to confirm that nothing came back. Abdominal radiographs were taken to confirm that the tip of the EFT was in the jejunum. This standardized procedure at our institution, which was based on a previously published protocol with minor modifications [21], had already been established several years before the first participants for our study were admitted to our ICU. The procedure was taught among inexperienced or novice physicians as following steps: (1) physicians took 
the lecture using the document about this procedure by instructors who were specialists of critical care; (2) they observed the procedure performed by instructors; (3) in the presence of instructors, the educated physicians performed the procedure several times. Abdominal radiographs were evaluated by the medical team, including specialists who have much experience with post-pyloric placement, and the findings, including the position of the EFT tip, were documented in the medical record. The decision to start enteral nutrition via post-pyloric EFT was decided by a consensus within a medical team. The enteral nutrition via the post-pyloric route in our institution was used as the first choice, in principle.

\section{Data acquisition}

The following 21 variables were extracted from electronic medical records, as candidate predictors: (1) age; (2) sex; (3) height; (4) body mass index (BMI); (5) fluid balance from admission to the procedure; (6) presence or absence of intestinal peristaltic movement; (7) serum albumin levels; (8) body position during the procedure; (9) position of the stomach; (10) Sequential Organ Failure Assessment (SOFA) score at the time of the procedure; (11) postoperative cardiovascular surgery; (12) blood disorders (leukemia, myelodysplastic syndrome, or malignant lymphoma); (13) respiratory diseases (acute respiratory distress syndrome, pneumonia, or acute exacerbation of chronic obstructive pulmonary disease or pulmonary fibrosis); (14) concurrent diabetes mellitus; (15) use of prokinetic agents; (16) use of sedatives; (17) opioid dosage; (18) use of vasopressor agents; (19) use of cardiac assist devices (intra-aortic balloon pumping, extra-corporeal membrane oxygenation, or ventricular assist device); (20) use of renal replacement therapy, and (21) ICU experience of the physician performing the procedure. BMI was calculated based on the patient's height and weight at ICU admission. Fluid balance was defined as an increase or decrease in body weight from ICU admission to the time of EFT placement. Albumin levels and SOFA scores were obtained from data collected closest to the time of EFT placement. The body position during the procedure was treated as a binary variable between right lateral and other positions. The position of the stomach was evaluated by the location of the greater curvature of the stomach relative to spinal level, as estimated by abdominal radiography after EFT placement; it was treated as a binary value of either cephalad or caudal to L1 $-\mathrm{L} 2$, which was the median value of our data (Fig. 1a, b). The amount of opioid used was converted to fentanyl dose per hour. The experience of the physician performing EFT placement was divided into four categories: junior residents, senior residents, fellows, and critical care specialists or supervisors. The presence

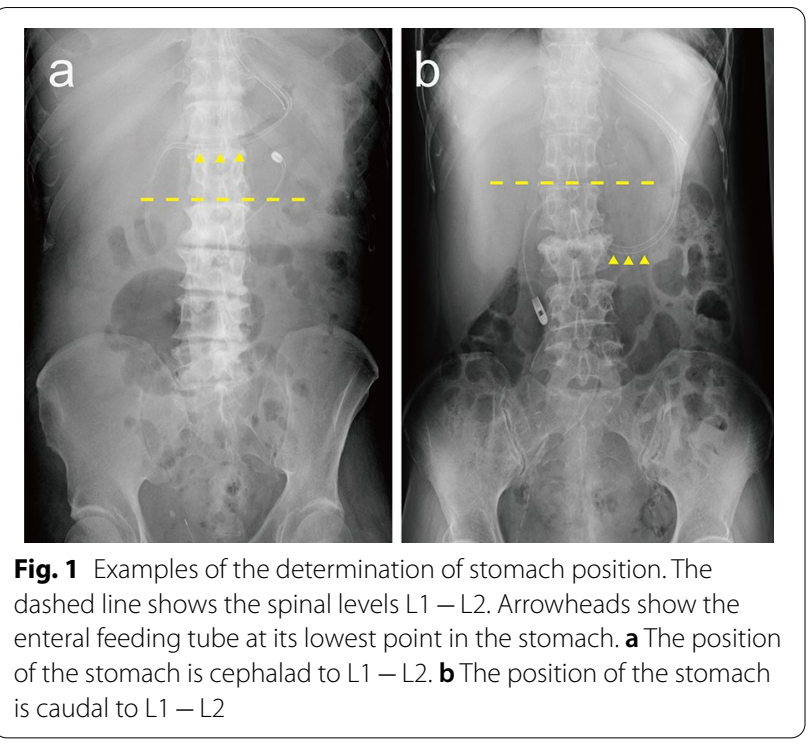

or absence of in-hospital mortality, ICU length of stay, ventilator-free days (VFD), and EFT-related outcomes (days from ICU admission to the start of enteral nutrition and days from EFT placement to the start of enteral nutrition) were extracted from the medical records. VFD was defined as 28 days minus the number of days with mechanical ventilation via tracheal intubation after ICU admission.

\section{Outcomes}

The primary outcome was the first-pass success rate of post-pyloric EFT placement. First-pass success was determined based on whether the EFT passed through the pylorus, as seen on the first abdominal radiograph after placement. If it was difficult to determine success or failure, it was comprehensively determined by two researchers who were involved in the research, referring to supplemental information such as descriptions in the medical records regarding the position of the EFT tip or the presence or absence of starting nutrition via EFT immediately after taking a first abdominal radiograph. The secondary outcomes were: (1) the correlation coefficient between the number of days from ICU admission to the start of enteral nutrition and the ICU length of stay; (2) the correlation between the number of days from EFT placement to the start of enteral nutrition and ICU length of stay, and (3) a description of patient outcomes.

\section{Sample size calculation}

The reported success rate of blind placement of postpyloric EFTs varies between 30 and 90\% [22-25]. In this study, we assumed that the success rate was $60 \%$, and 18 factors were selected as independent variables for logistic 
regression analysis. As the adopted number of negative events per variable was 10 , the total number of negative events required was 180 (i.e., multiplying 18 variables by 10). Therefore, we calculated 450 as the minimum number of patients required.

\section{Statistical analyses}

All data are expressed as median (interquartile range) or numbers (percentages), as appropriate. Logistic regression analysis was performed to assess the association between first-pass success and the independent variables. We selected variables with a $P<0.2$ in the univariate analysis, and variables expected to be involved in the first-pass success rate based on the clinical perspective, as the independent variables. In the univariate analysis, the unpaired $t$-test, Mann-Whitney $U$ test, or Fisher's exact test was performed, as appropriate. All parameters were checked for multicollinearity. We performed complete data analysis to address missing values. Correlation coefficients for secondary outcomes were assessed using
Spearman's rank correlation. For all analyses, a two-tailed $P<0.05$ was considered statistically significant. All statistical analyses were performed using Microsoft Excel 2016 (Microsoft; Redmond, WA, USA) and R software (version 3.3.2: R Foundation for Statistical Computing; Vienna, Austria).

\section{Results}

A total of 455 patients were included in this study. After 13 patients were excluded (10 patients, EFT was inserted before ICU admission; three patients, a history of upper gastrointestinal surgery), we analyzed data from 442 patients who met the inclusion criteria (Fig. 2). Table 1 shows the patient characteristics. The median age and SOFA score were $68(57-76)$ years and $10(7-13)$, respectively. The percentage of men was $60 \%$. The firstpass success rate of blind placement of a post-pyloric EFT was $42.8 \%(n=189)$. The median ICU length of stay and VFD was $8(6-14)$ days and $21(6-24)$ days,

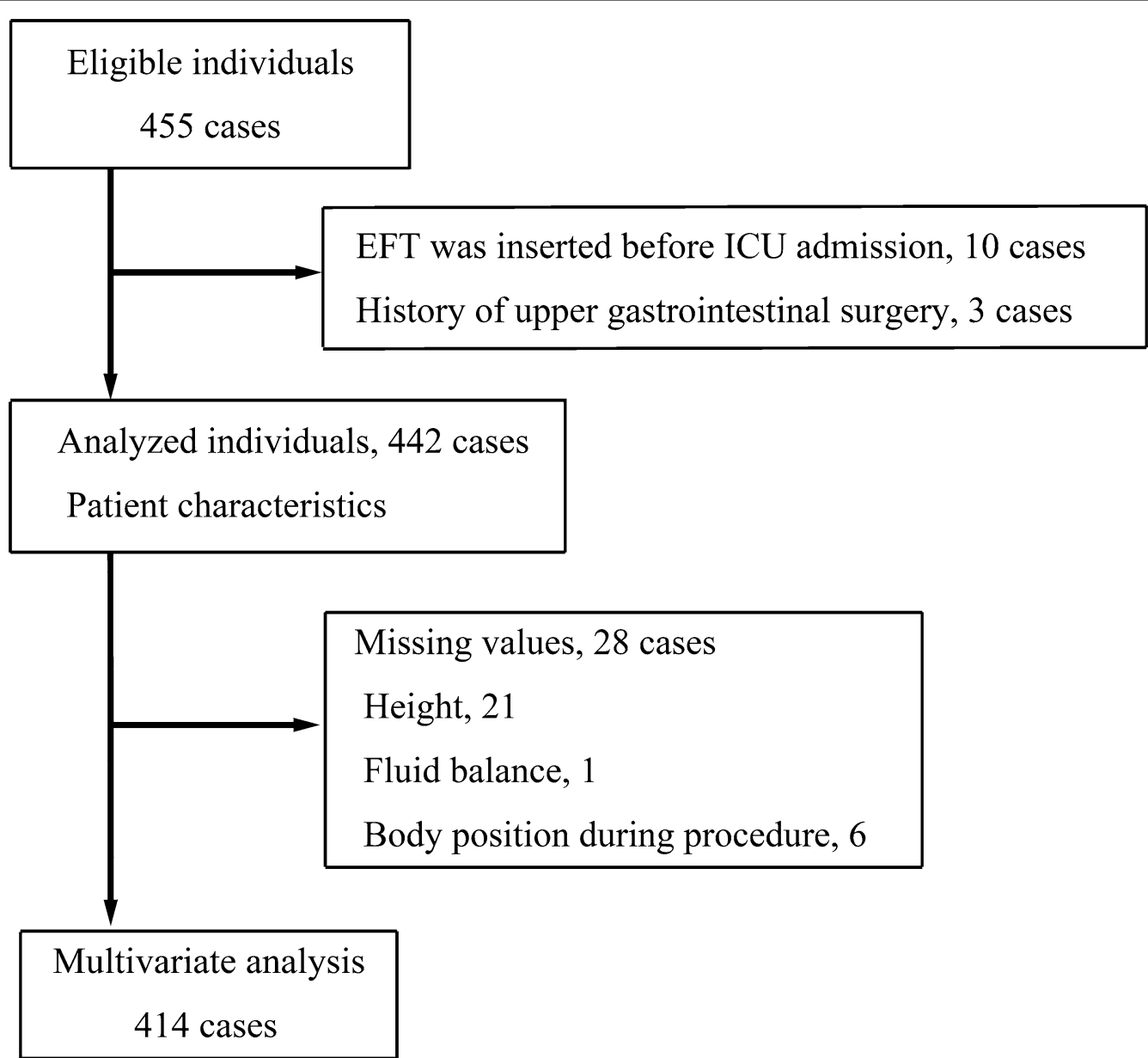

Fig. 2 Study flowchart. EFT enteral feeding tube; ICU intensive care unit 
Table 1 Patient characteristics and results of the univariate analysis

\begin{tabular}{|c|c|c|c|c|}
\hline Characteristic & Overall $(n=442)$ & Success $(n=189)$ & Failure $(n=253)$ & $P$-value \\
\hline Age (years) & $68(57-76)$ & $67(54-75)$ & $68(59-77)$ & 0.19 \\
\hline \multicolumn{5}{|l|}{ Sex, $n(\%)$} \\
\hline Male & $265(60)$ & $103(23)$ & $162(37)$ & \multirow[t]{2}{*}{0.049} \\
\hline Female & $177(40)$ & $86(19)$ & $91(21)$ & \\
\hline Height (cm) & $162(154-168)$ & $160(153-168)$ & $162(155-168)$ & 0.20 \\
\hline Weight (kg) & $58(48-66)$ & $58(48-65)$ & $58(50-67)$ & 0.45 \\
\hline Body mass index $\left(\mathrm{kg} / \mathrm{m}^{2}\right)$ & $22.4(19.8-24.9)$ & $22.4(19.8-24.7)$ & $22.4(19.8-25.2)$ & 0.92 \\
\hline Fluid balance from baseline $(\mathrm{kg})$ & $2.3(0.4-4.8)$ & $2.5(0.6-0.9)$ & $2.1(0.2-4.6)$ & 0.30 \\
\hline SOFA score & $10(7-13)$ & $10(7-12)$ & $10(7-13)$ & 0.87 \\
\hline Serum albumin (g/dL) & $2.7(2.3-3.1)$ & $2.7(2.2-3.1)$ & $2.8(2.3-3.2)$ & 0.12 \\
\hline Position of the stomach & L1 - L2 (T12 - L1 to L2 - L3) & L1 - L2 (T12 - L1 to L2 - L3) & L1 - L2 (T12- L1 to L2 - L3) & 0.06 \\
\hline \multicolumn{5}{|l|}{ Use of sedatives, $n(\%)$} \\
\hline No & $49(11)$ & $25(13)$ & $24(9)$ & \multirow[t]{2}{*}{0.22} \\
\hline Yes & $393(89)$ & $164(87)$ & $229(91)$ & \\
\hline Use of a vasopressor, $n(\%)$ & $307(69)$ & $132(70)$ & $175(69)$ & 0.92 \\
\hline Fentanyl dose ( $\mu \mathrm{g} / \mathrm{hour})$ & $20(10-20)$ & $20(0-20)$ & $20(10-25)$ & 0.38 \\
\hline Right lateral position, n (\%) & 169 (38) & $75(40)$ & $94(37)$ & 0.69 \\
\hline Use of a prokinetic agent, $n(\%)$ & $22(5)$ & $11(6)$ & $11(4)$ & 0.51 \\
\hline Presence of intestinal peristalsis, $n(\%)$ & $116(26)$ & $58(30)$ & $58(23)$ & 0.08 \\
\hline \multicolumn{5}{|l|}{ Degree of experience, $n(\%)$} \\
\hline Junior resident & $84(19)$ & $34(18)$ & $50(20)$ & \multirow[t]{5}{*}{0.45} \\
\hline Senior resident & $47(11)$ & $23(12)$ & $24(10)$ & \\
\hline Fellow & $107(24)$ & $41(22)$ & $66(26)$ & \\
\hline Specialist & $21(5)$ & $10(5)$ & $11(4)$ & \\
\hline Missing values & $183(41)$ & $81(43)$ & $102(40)$ & \\
\hline Diabetes mellitus, n (\%) & $92(21)$ & $36(19)$ & $56(22)$ & 0.48 \\
\hline Renal replacement therapy, $n$ (\%) & $50(11)$ & $16(8)$ & $34(13)$ & 0.13 \\
\hline Use of cardiac assist devices, $n$ (\%) & $36(8)$ & $13(7)$ & $23(9)$ & 0.48 \\
\hline Post-cardiac surgery, $n(\%)$ & $180(41)$ & $69(37)$ & $111(44)$ & 0.14 \\
\hline Hematology, n (\%) & $43(10)$ & $20(11)$ & $23(9)$ & 0.63 \\
\hline Respiratory disease, $n$ (\%) & $114(26)$ & $52(28)$ & $62(25)$ & 0.51 \\
\hline
\end{tabular}

Body mass index was calculated based on the height and weight at intensive care unit (ICU) admission. Cardiac assist devices included intra-aortic balloon pumping, extra-corporeal membrane oxygenation, and ventricular assist devices. Fluid balance was defined as an increase or decrease in body weight from ICU admission to enteral feeding tube placement. Hematology included patients with blood disorders, such as leukemia, myelodysplastic syndrome, and malignant lymphoma. Post-cardiac surgery included patients admitted to the ICU for postoperative management of cardiovascular surgery. Respiratory disease included patients with acute respiratory distress syndrome, pneumonia, and acute exacerbation of chronic obstructive pulmonary disease or pulmonary fibrosis. Position of the stomach was defined as the position of the greater curvature of the stomach caudal or cephalad to L1-L2, as estimated by abdominal radiography. Weight was defined as body weight before ICU admission. All values are expressed as number (percentage) or median (interquartile range). SOFA Sequential Organ Failure Assessment

respectively. The number of patients who died in the ICU was 35 (8\%) (Table 2).

The eight variables with $P<0.2$ in the univariate analysis were: age, sex, height, serum albumin levels, position of the stomach, presence of intestinal peristaltic movement, renal replacement therapy, and post-cardiovascular surgery (Table 1). In addition, five factors were selected as variables expected to be involved in the first-pass success rate based on the clinical perspective: body position during placement, use of prokinetic agents, fluid balance, use of cardiac assist devices, and use of sedatives. Because multicollinearity was not found in these 13 variables, all variables were included in the final analysis. Twentyeight cases with missing values (21 cases, height; one case, fluid balance; and six cases, body position during EFT placement) were excluded from the final analysis. The results of the logistic regression analysis with these 13 factors as independent variables are shown in Table 3.

A stomach position caudal to $\mathrm{L} 1-\mathrm{L} 2$ was associated with a lower first-pass success rate (adjusted odds ratio, $0.61 ; 95 \%$ confidence interval: $0.40-0.94 ; P=0.03$ ). The number of days from ICU admission to the start of 
Table 2 Summary of outcome data

\begin{tabular}{lccc}
\hline Outcome & Overall $(\boldsymbol{n = 4 4 2 )}$ & Success $(\boldsymbol{n = 1 8 9})$ & Failure $(\boldsymbol{n}=\mathbf{2 5 3})$ \\
\hline Death in the ICU, $n$ (\%) & $35(8)$ & $18(4)$ & $17(4)$ \\
Death in the hospital, $n(\%)$ & $100(23)$ & $42(10)$ & $58(13)$ \\
Death after ICU admission (days) & $27(13-60)$ & $34(14-70)$ & $23(12-42)$ \\
Ventilator-free days & $21(6-24)$ & $20(5-24)$ & $21(6-24)$ \\
Time from EFT placement to the start of enteral & $1(0-2)$ & $0(0-1)$ & $1(1-2)$ \\
feeding (days) & $2(1-4)$ & $8(1-3)$ & $3(2-4)$ \\
Time from ICU admission to the start of enteral & & $8(6-15)$ & $8(5-13)$ \\
feeding (days) & $8(6-14)$ &
\end{tabular}

Death in the hospital was defined as the number of patients who died in the ICU or on the ward. Ventilator-free days were calculated as 28 days minus the number of days without mechanical ventilation via tracheal intubation. All values are expressed as number (percentage) or median (interquartile range). EFT enteral feeding tube, ICU intensive care unit

Table 3 Multivariate analysis of the first-pass success rate of enteral feeding tube placement

\begin{tabular}{llll}
\hline Variable & Odds ratio & $\mathbf{9 5 \%} \mathbf{C l}$ & P-value \\
\hline Age (each 10-year increment) & 0.91 & $0.78-1.06$ & 0.22 \\
Sex (female) & 1.32 & $0.74-2.37$ & 0.35 \\
Height (each 10-cm increment) & 0.91 & $0.68-1.23$ & 0.55 \\
Fluid balance (each 1-kg increment) & 1.04 & $0.98-1.10$ & 0.18 \\
Serum albumin level (each 1-g/dL increment) & 0.74 & $0.50-1.07$ & 0.11 \\
Position of the stomach (caudal to L1 - L2) & 0.61 & $0.42-1.55$ & 0.04 \\
Use of sedatives & 0.80 & $0.66-1.53$ & 0.51 \\
Right lateral body position & 1.01 & $0.47-3.07$ & 0.97 \\
Use of prokinetic agents & 1.20 & $0.47-1.20$ & $0.26-1.05$ \\
Presence of intestinal peristaltic movement & 0.75 & $0.39-2.01$ & 0.23 \\
Renal replacement therapy & 0.52 & $0.58-1.60$ & 0.07 \\
Use of cardiac assist devices & 0.86 & 0.70 & 0.87 \\
Post-cardiovascular surgery & 0.96 &
\end{tabular}

Factors with an odds ratio $>1.0$ are associated with successful EFT placement. $\mathrm{Cl}$ confidence interval

enteral nutrition was $2(1-4)$ days. The correlation coefficient between the number of days from ICU admission to the start of enteral nutrition and ICU length of stay was $0.22(P<0.001)$. The median number of days from ICU admission to the start of enteral nutrition in patients with first-pass success was lower than that in those without first-pass success. The absolute difference was 1 day (first-pass success vs. failure: $2[1-3]$ vs. 3 [2-4] days) (Table 2). The correlation coefficient between the number of days from EFT placement to the start of enteral nutrition and ICU length of stay was $0.15(P<0.001)$. The median ICU length of stay was 8 days in both the firstpass success and failure groups (Table 2).

\section{Discussion}

We demonstrated that a position of the greater curvature of the stomach lower than spinal level L1 - L2 may be associated with a lower first-pass success rate of blind placement of a post-pyloric EFT in critically ill patients. Notably, the stomach position was evaluated using abdominal radiography after EFT placement as part of the clinical procedures to confirm correct EFT placement. However, because the position of the stomach was estimated after EFT placement, whether the stomach position before EFT placement is associated with the success of blind tube placement is unknown.

Our study was not designed to elucidate the mechanism(s) underlying our findings; however, several possibilities warrant consideration. First, a more caudal position of the greater curvature of the stomach results in a more angulated path from the gastric inlet (the cardia) to the outlet (the pylorus), which is more difficult for the EFT to travel along. Second, a more caudal position of the greater curvature of the stomach may indicate greater extensibility of the stomach. Gastric extensibility varies greatly among individuals [26, 27]. A more extensible stomach may have absorbed the incremental 
advancement of the EFT instead of guiding it in the direction of the pylorus, resulting in failure of the tube to pass through the pylorus. Third, a gastric extension may also have extended the length of the EFT required for pyloric passage. Therefore, there may have been cases where the EFT did not reach the pylorus because the physicians may have been hesitant to insert the EFT beyond the depth of their empirical predictions.

The reported success rate of post-pyloric EFT placement varies greatly $[22-25,28,29]$. Our success rate was $42.8 \%$, which is unfavorable compared to previous studies. This may be explained by the subtle differences in detailed placement procedures/protocols or by differences in the study populations, such as the inclusion or exclusion of patients with circulatory instability [28] or a high BMI [29]. Our study population, which included these patients, may reveal a success rate in a patient population with higher severity. Conversely, in our retrospective study, the lack of assessment of the adherence rate to our institutional protocol for EFT placement procedures may be a disadvantage for generalization, because a lower adherence rate may be associated with a lower success rate. In our ICU, care was taken to ensure the quality of the placement procedures when performed by inexperienced staff by having more experienced staff supervise.

A recent study suggested that several factors, including the severity of the participants' condition (Acute Physiology and Chronic Health Evaluation II score $\geq 20$, SOFA score $\geq 12$, use of vasopressor, and patients with mechanical ventilation), patients with neurological diseases, use of continuous renal replacement therapy, and impaired gastrointestinal function, may be associated with the success rate of blind tube placement [20]. Elucidation of risk factors may provide additional information for the strategy of nutrition therapy in critically ill patients. However, further evidence will need to be accumulated because the settings in the studies reported previously are limited.

Our study could not elucidate the effect of prokinetic agents due to the wide confidence interval caused by the small number of patients who received them. A previous meta-analysis showed that the use of prokinetics in patients without intestinal peristalsis promoted successful EFT placement [30]. However, the Cochrane review in 2015 argued that a higher-quality, large randomized controlled study is necessary to examine this further [31].

Our study had several limitations. First, this was a retrospective, single-center study and, therefore, could not be adjusted for unknown confounders, and external validity may be limited. Second, the confidence intervals of odds ratios for several variables were wide, partly because of the small number of positive cases. Third, the experience levels of the physicians performing the EFT placements could not be evaluated because there was too much missing data. It was difficult to quantitatively evaluate the experience level of the physicians with high accuracy in retrospective data obtained from the medical record. We excluded this factor from the final analysis because: first, we were concerned that the reduction in the number of cases might have made it difficult to achieve our goal of a comprehensive search for associated factors. Second, in our ICU, the quality of the procedures was ensured by the supervision of senior physicians. Additional logistic regression analysis, which was a sensitivity analysis including experience levels of the physician, confirmed that the position of the stomach, which was the main result, had a similar direction of effect (data not shown). Finally, first-pass success or failure of EFT placement which was our primary outcome, might include the bias because it was estimated by the investigators associated with the study.

In this study, the position of the stomach was estimated after, but not before, EFT placement. Therefore, our results cannot be used to identify predictive factors before EFT placement. The main objective of this study was to generate hypotheses through a comprehensive investigation of factors associated with first-pass success. Therefore, we used the abdominal radiographs after EFT placement simply as an index of the position of the stomach because it is our routine clinical practice to confirm the position of the EFT by abdominal radiographs after the placement. Because our clinical practice does not require pre-placement abdominal radiographs or computed tomography, we were afraid that incorporation of the pre-placement abdominal radiographic examination into our study design would reduce the number of cases that could be enrolled, leading to insufficient statistical power for multivariate analysis. Further studies are needed to determine whether a lower stomach position on abdominal radiograph or computed tomography before EFT placement can predict the difficulty of EFT placement.

\section{Conclusions}

The greater curvature of the stomach caudal to spinal level L1-L2 may be associated with the first-pass failure of post-pyloric EFT placement. Further studies with larger numbers of patients are needed to verify whether the lower stomach position before EFT placement can predict the difficulty of EFT placement.

\section{Abbreviations}

BMI: Body mass index; Cl: Confidence interval; EFT: Enteral feeding tube; ICU: Intensive care unit; SOFA: Sequential Organ Failure Assessment; VFD: Ventilator-free days. 


\section{Authors' information}

Kohei Kurisawa and Masashi Yokose contributed equally to this manuscript as co-first authors.

\section{Acknowledgements}

Not applicable.

\section{Authors' contributions}

KK drafted the manuscript and collected the data. MY drafted the manuscript and collected, analyzed, and interpreted the data. HT collected the data and revised the manuscript critically for important intellectual content. MY, TM and ST contributed to the conception and design of the study. TM analyzed and interpreted the data and revised the manuscript critically for important intellectual content. ST and TG revised the manuscript critically for important intellectual content and supervised the study. All authors read and approved the final manuscript.

\section{Funding}

This research did not receive any specific grant from funding agencies in the public, commercial, or not-for-profit sectors.

\section{Availability of data and materials}

The datasets used and/or analyzed during the current study are available from the corresponding author on reasonable request.

\section{Declarations}

\section{Ethics approval and consent to participate}

The study was approved by the Ethics Board of Yokohama City University Hospital (approval number: B181000027; November 22, 2018), which approved an opt-out method of informed consent. The trial was registered with the University Medical Information Network Clinical Trials Registry (UMIN000036549; April 19, 2019) before data collection.

\section{Consent for publication}

Not applicable.

\section{Competing interests}

The authors declare that they have no competing interests.

\section{Author details}

${ }^{1}$ Department of Anesthesiology and Critical Care Medicine, Yokohama City University Graduate School of Medicine, 3-9 Fukuura, Kanazawa-ku, Yokohama 236-0004, Japan. ${ }^{2}$ Department of Health Data Science, Yokohama City University Graduate School of Data Science, Yokohama, Japan.

Received: 1 August 2021 Accepted: 30 September 2021

Published online: 07 October 2021

\section{References}

1. Lew CCH, Yandell R, Fraser RJL, Chua AP, Chong MFF, Miller M. Association between malnutrition and clinical outcomes in the Intensive Care Unit: a systematic review. JPEN J Parenter Enteral Nutr. 2017:41:744-58.

2. Ali NA, O'Brien JM, Hoffmann SP, Phillips G, Garland A, Finley JCW, et al. Acquired weakness, handgrip strength, and mortality in critically ill patients. Am J Respir Crit Care Med. 2008;178:261-8.

3. Mogensen KM, Robinson MK, Casey JD, Gunasekera NS, Moromizato T, Rawn JD, et al. Nutritional status and mortality in the critically ill. Crit Care Med. 2015:43:2605-15.

4. Schneider SM, Veyres P, Pivot X, Soummer AM, Jambou P, Filippi J, et al. Malnutrition is an independent factor associated with nosocomial infections. Br J Nutr. 2004;92:105-11.

5. Giner M, Laviano A, Meguid MM, Gleason JR. In 1995 a correlation between malnutrition and poor outcome in critically ill patients still exists. Nutrition. 1996;12:23-9.

6. Japanese guidelines for nutrition support therapy in the adult and pediatric critically ill patients. J Japanese Soc Intensive Care Med. 2016:23:185-281.
7. Elke G, Van Zanten ARH, Lemieux M, Mccall M, Jeejeebhoy KN, Kott $M$, et al. Enteral versus parenteral nutrition in critically ill patients: an updated systematic review and meta-analysis of randomized controlled trials. Crit Care. 2016;20:117

8. Peter JV, Moran JL, Phillips-Hughes J. A metaanalysis of treatment outcomes of early enteral versus early parenteral nutrition in hospitalized patients. Crit Care Med. 2005:33:213-20.

9. Harvey SE, Parrott F, Harrison DA, Sadique MZ, Grieve RD, Canter RR, et al. A multicentre, randomised controlled trial comparing the clinical effectiveness and cost-effectiveness of early nutritional support via the parenteral versus the enteral route in critically ill patients (CALORIES). Health Technol Assess. 2016;20:1-144.

10. Sadique Z, Harrison DA, Bear DE, Rowan KM, Grieve R, CALORIES trial investigators. Effectiveness and cost-effectiveness of early nutritional support via the parenteral versus the enteral route for critically ill adult patients. J Crit Care. 2019;52:237-41.

11. Kang W, Kudsk KA. Is there evidence that the gut contributes to mucosal immunity in humans? JPEN J Parenter Enteral Nutr. 2007;31:246-58.

12. Gianotti L, Alexander JW, Nelson JL, Fukushima R, Pyles T, Chalk CL. Role of early enteral feeding and acute starvation on postburn bacterial translocation and host defense: prospective, randomized trials. Crit Care Med. 1994;22:265-72.

13. Kudsk KA. Current aspects of mucosal immunology and its influence by nutrition. Am J Surg. 2002:183:390-8.

14. McClave SA, Taylor BE, Martindale RG, Warren MM, Johnson DR, Braunschweig $C$, et al. Guidelines for the provision and assessment of nutrition support therapy in the adult critically ill patient: Society of Critical Care Medicine (SCCM) and American Society for Parenteral and Enteral Nutrition (ASPEN). JPEN J Parenter Enteral Nutr. 2016:40:159-211.

15. Alhazzani W, Almasoud A, Jaeschke R, Lo BW, Sindi A, Altayyar S, et al. Small bowel feeding and risk of pneumonia in adult critically ill patients: a systematic review and meta-analysis of randomized trials. Crit Care. 2013:17:R127.

16. Jiyong J, Tiancha $H$, Huiqin W, Jingfen J. Effect of gastric versus postpyloric feeding on the incidence of pneumonia in critically ill patients: observations from traditional and Bayesian random-effects meta-analysis. Clin Nutr. 2013;32:8-15.

17. Fang JC, Hilden K, Holubkov R, DiSario JA. Transnasal endoscopy vs. fluoroscopy for the placement of nasoenteric feeding tubes in critically ill patients. Gastrointest Endosc. 2005;62:661-6.

18. Thurley PD, Hopper MA, Jobling JC, Teahon K. Fluoroscopic insertion of post-pyloric feeding tubes: success rates and complications. Clin Radiol. 2008;63:543-8

19. Wiggins TF, DeLegge MH. Evaluation of a new technique for endoscopic nasojejunal feeding-tube placement. Gastrointest Endosc. 2006;63:590-5.

20. Wang Q, Xuan Y, Liu C, Lu M, Liu Z, Chang P. Blind placement of postpyloric feeding tubes at the bedside in intensive care. Crit Care. 2021;25:168.

21. Okutani K, Hayami H, Ohki H, Kokawa A, Nagai S, Ohtsuka M, et al. A simple technique for bedside insertion of trans pyloric enteral feeding tubes; without special devices or drugs. J Japanese Soc Intensive Care Med. 2007:14:177-85.

22. Marik PE, Zaloga GP. Gastric versus post-pyloric feeding: a systematic review. Crit Care 2003:7:R46-51.

23. Lai CWY, Barlow R, Barnes M, Hawthorne AB. Bedside placement of nasojejunal tubes: a randomised-controlled trial of spiral- vs straight-ended tubes. Clin Nutr. 2003;22:267-70.

24. Lenart S, Polissar NL. Comparison of 2 methods for postpyloric placement of enteral feeding tubes. Am J Crit Care. 2003;12:357-60.

25. Hu B, Ye H, Sun C, Zhang Y, Lao Z, Wu F, et al. Metoclopramide or domperidone improves post-pyloric placement of spiral nasojejunal tubes in critically ill patients: a prospective, multicenter, open-label, randomized, controlled clinical trial. Crit Care. 2015;19:61.

26. Standring S. Gray's anatomy: the anatomical basis of clinical practice Gray's anatomy. 40th ed. Edinburgh: Elsevier Churchill Livingstone; 2008.

27. Natsis K, Apostolidis S, Papadopoulou AL, Vlasis K, Totlis T, Skandalakis P. Gastric femoral hernia in a male cadaver with gastroptosis: case report and review of the literature. Hernia. 2008;12:205-8.

28. Griffith DP, McNally AT, Battey CH, Forte SS, Cacciatore AM, Szeszycki EE, et al. Intravenous erythromycin facilitates bedside placement of postpyloric feeding tubes in critically ill adults: a double-blind, randomized, placebo-controlled study. Crit Care Med. 2003;31:39-44. 
29. Kohata H, Okuda N, Nakataki E, Itagaki T, Onodera M, Imanaka H, et al. A novel method of post-pyloric feeding tube placement at bedside. J Crit Care. 2013;28:1039-41.

30. Tiancha H, Jiyong J, Min Y. How to promote bedside placement of the postpyloric feeding tube: a network meta-analysis of randomized controlled trials. JPEN J Parenter Enteral Nutr. 2015;39:521-30.

31. Silva CC, Bennett C, Saconato H, Atallah ÁN. Metoclopramide for postpyloric placement of naso-enteral feeding tubes. Cochrane Database Syst Rev. 2015. https://doi.org/10.1002/14651858.CD003353.pub2.

\section{Publisher's Note}

Springer Nature remains neutral with regard to jurisdictional claims in published maps and institutional affiliations.
Ready to submit your research? Choose BMC and benefit from:

- fast, convenient online submission

- thorough peer review by experienced researchers in your field

- rapid publication on acceptance

- support for research data, including large and complex data types

- gold Open Access which fosters wider collaboration and increased citations

- maximum visibility for your research: over $100 \mathrm{M}$ website views per year

At BMC, research is always in progress.

Learn more biomedcentral.com/submissions 